\title{
Momentum constraint relaxation
}

\author{
Pedro Marronetti \\ Department of Physics, Florida Atlantic University, Boca Raton, FL 33431
}

\begin{abstract}
Full relativistic simulations in three dimensions invariably develop runaway modes that grow exponentially and are accompanied by violations of the Hamiltonian and momentum constraints. Recently, we introduced a numerical method (Hamiltonian relaxation) that greatly reduces the Hamiltonian constraint violation and helps improve the quality of the numerical model. We present here a method that controls the violation of the momentum constraint. The method is based on the addition of a longitudinal component to the traceless extrinsic curvature $\tilde{A}_{i j}$, generated by a vector potential $w_{i}$, as outlined by York. The components of $w_{i}$ are relaxed to solve approximately the momentum constraint equations, pushing slowly the evolution toward the space of solutions of the constraint equations. We test this method with simulations of binary neutron stars in circular orbits and show that effectively controls the growth of the aforementioned violations. We also show that a full numerical enforcement of the constraints, as opposed to the gentle correction of the momentum relaxation scheme, results in the development of instabilities that stop the runs shortly.
\end{abstract}

PACS numbers: 04.30.Db, 04.25.Dm, 97.80.Fk

E-mail: pmarrone@physics.fau.edu 


\section{Introduction}

For most of the past decade, the main theoretical thrust in gravitational research has been directed toward obtaining stable and accurate numerical models of compact-object binary systems. One of the most difficult problems to tackle has been the control of exponentially growing instabilities that degrade the quality of any simulation and, eventually, terminate it. General relativistic numerical simulations of BNS systems have made significant progress in the past years [1, 2, 3, 4, 5, 6, 7], in particular with the addition of advanced numerical techniques such as AMR [8].

The numerical formulations employed to simulate astrophysical systems can be divided in unconstrained and constrained methods. Unconstrained formulations like ADM [9] and BSSN [10, 11] simply evolve the gravitational fields without any attempts at controlling the violation of the time independent Hamiltonian and momentum constraints; these equations are merely monitored to gauge the accuracy of the model. Constrained formalisms, on the other hand, enforce the satisfaction of the constraint equations at either the analytical level (i.e., ingraining them in some way in the time evolution equations) or at the numerical level (i.e., regularly solving the constraints during the evolution). While constrained methods have been applied mostly to problems with spherical [12] and axial symmetry [13, 14, 15], there have been recent applications to three-dimensional scenarios [16, 17].

We propose in this and a companion article [18] (from now on, Paper I) an alternative type of evolution in which the Hamiltonian and momentum constraints are only approximately solved at every time step, gently steering the evolution toward the space of solutions of these equations without completely forcing their numerical satisfaction. In Paper I we described a method that controls the Hamiltonian constraint violation (Hamiltonian relaxation or $\mathrm{HR}$ ). In this paper we introduce a complementary scheme that reduces the momentum constraint residuals (momentum relaxation or MR). The constraint relaxation techniques utilize the conformal decomposition of the spatial metric and extrinsic curvature presented by York [19, 20], which has traditionally been used to solve the initial value problem for binary systems [21, 22, 23, 24, 25]. In this decomposition, a conformal factor $\psi$ factored out of the spatial metric and a longitudinal addition to the extrinsic curvature generated from a vector potential $w_{i}$ are used to satisfy the Hamiltonian and the three components of the momentum constraint respectively. HR drives $\psi$ to the solution space of the Hamiltonian constraint by means of a parabolic equation for the conformal factor, in the spirit of the K-Driver [26] used for the lapse. The MR method described in this paper uses $w_{i}$ to push the simulation toward the space of solutions of the momentum constraint. In both cases, a full relaxation of $\psi$ and $w_{i}$ would lead to the numerical solution of the constraints. However, the stability of the relaxation methods relies on gently updating these fields during the evolution. We show in Appendix A that a full relaxation scheme becomes unstable rather quickly when used in combination with BSSN.

We tested the new algorithms by simulating binary neutron stars (BNS) in circular 
orbits and show that the use of these techniques results in a notable improvement of the overall quality of the simulation. HR not only suppresses the Hamiltonian constraint violation but also contributes to a more stable behavior in the total angular momentum of the system. MR contribution is mostly confined to quenching the momentum constraint violation. The BNS simulation runs for about two orbits before stopping. One of the main reasons for the degradation of the simulation quality is the use of a shift vector frozen to its initial value ( $\beta$-freeze). This choice, while very convenient when testing new algorithms, becomes inadequate as soon as the stars move appreciably from their initial position. Given that our simulations are performed in the frame that rotates with the binary, this occurs rather late in the run. The second cause is related to inappropriate boundary conditions for the rest of the gravitational fields. The use of radiation (Sommerfeld) conditions in rotating frames becomes troublesome for large grids in rotating frames. These problems will be addressed in future work.

Section 2 describes in detail the momentum constraint relaxation method and its numerical implementation. Section 3 presents simulations of BNS in circular orbits and compares the results obtained with and without the relaxation techniques. Section 4 summarizes our results and Appendix A presents the convergence tests.

\section{Equations for the Gravitational and Hydrodynamical Fields}

\subsection{Time Evolution Equations}

We use geometrized units $(G=c=1)$ and the Greek (Latin) indices run from 0 to 3 (1 to 3$)$. In the standard " $3+1$ " form, the metric is written as

$$
\mathrm{d} s^{2}=-\alpha^{2} \mathrm{~d} t^{2}+\gamma_{i j}\left(\mathrm{~d} x^{i}+\beta^{i} \mathrm{~d} t\right)\left(\mathrm{d} x^{j}+\beta^{j} \mathrm{~d} t\right)
$$

where $\alpha, \beta^{i}$, and $\gamma_{i j}$ are the lapse function, shift vector, and spatial metric tensor, respectively.

The ADM formulation [9] splits the Einstein's field equations

$$
G_{\mu \nu}=8 \pi T_{\mu \nu}
$$

into a set of time-independent elliptic equations

$$
\begin{aligned}
& R-K_{i j} K^{i j}+K^{2}=16 \pi \rho, \\
& D_{j} K_{i}^{j}-D_{i} K=8 \pi S_{i},
\end{aligned}
$$

known as the Hamiltonian and momentum constraints, plus a set of time-dependent hyperbolic equations:

$\left(\partial_{t}-\mathcal{L}_{\beta}\right) \gamma_{i j}=-2 \alpha K_{i j}$,

$\left(\partial_{t}-\mathcal{L}_{\beta}\right) K_{i j}=-D_{i} D_{j} \alpha+\alpha\left\{R_{i j}-2 K_{i l} K^{l}{ }_{j}+K K_{i j}-8 \pi\left[S_{i j}+\frac{1}{2} \gamma_{i j}(\rho-S)\right]\right\}$.

The latter set provides the evolution in time of the spatial metric $\gamma_{i j}$ and the extrinsic curvature $K_{i j}$. The symbol $D_{i}$ represents the covariant gradient with respect to the tensor $\gamma_{i j}$. The fields $\rho, S$, and $S_{i j}$ are derived from the matter fields by splitting the 
stress-energy tensor $T_{\mu \nu}$ in components parallel and perpendicular to the normal of the spatial hypersurface $n^{\alpha}[18$.

Following York [19, 20], we can decompose the tensors $\gamma_{i j}$ and $K_{i j}$ as

$$
\begin{aligned}
& \gamma_{i j}=\psi^{4} \tilde{\gamma}_{i j}, \\
& K_{i j}=\psi^{4}\left(\tilde{A}_{i j}+\frac{1}{3} \tilde{\gamma}_{i j} K\right),
\end{aligned}
$$

Where the fields $\psi, \tilde{\gamma}_{i j}, \tilde{A}_{i j}$, and $K$, are known as the conformal factor, the conformal metric, the conformal traceless extrinsic curvature, and the trace of the extrinsic curvature respectively. We can write the Hamiltonian and momentum constraints using the new variables as

$$
\begin{aligned}
& \tilde{\gamma}^{i j} \tilde{D}_{i} \tilde{D}_{j} \psi-\frac{\psi}{8} \tilde{R}+\frac{\psi^{5}}{8} \tilde{A}_{i j} \tilde{A}^{i j}-\frac{\psi^{5}}{12} K^{2}+2 \pi \psi^{5} \rho=0, \\
& \tilde{D}^{j}\left(\psi^{6} \tilde{A}_{j i}\right)-\frac{2}{3} \psi^{6} \tilde{D}_{i} K-8 \pi \psi^{6} S_{i}=0 .
\end{aligned}
$$

We define the Hamiltonian constraint residual $\mathcal{H}$ and momentum constraint residual $\mathcal{M}_{i}$ as the 1.h.s. of equations (5) and (6) respectively.

The BSSN formulation [10, 11] provides a set of evolution equations for the fields $\psi, \tilde{\gamma}_{i j}, K$, and $\tilde{A}_{i j}$

$$
\begin{aligned}
& \left(\partial_{t}-\mathcal{L}_{\beta}\right) \ln (\psi)=-\frac{1}{6} \alpha K, \\
& \left(\partial_{t}-\mathcal{L}_{\beta}\right) \tilde{\gamma}_{i j}=-2 \alpha \tilde{A}_{i j}, \\
& \left(\partial_{t}-\mathcal{L}_{\beta}\right) K=-\gamma^{i j} D_{j} D_{i} \alpha+\frac{1}{3} \alpha K^{2}+\alpha \tilde{A}_{i j} \tilde{A}^{i j}+4 \pi \alpha(\rho+S), \\
& \left(\partial_{t}-\mathcal{L}_{\beta}\right) \tilde{A}_{i j}=\psi^{-4}\left[-D_{i} D_{j} \alpha+\alpha\left(R_{i j}-8 \pi S_{i j}\right)\right]^{T F}+\alpha\left(K \tilde{A}_{i j}-2 \tilde{A}_{i l} \tilde{A}_{j}^{l}\right),
\end{aligned}
$$

where the superscript $T F$ indicates the trace-free part of the tensor. These fields are complemented with the variable known as the conformal connection

$$
\tilde{\Gamma}^{i} \equiv-\tilde{\gamma}^{i j}{ }_{j},
$$

and its corresponding evolution equation

$$
\begin{aligned}
\partial_{t} \tilde{\Gamma}^{i} & =\partial_{j}\left(2 \alpha \tilde{A}^{i j}+\mathcal{L}_{\beta} \tilde{\gamma}^{i j}\right) \\
& =\tilde{\gamma}^{j k} \beta^{i}{ }_{, j k}+\frac{1}{3} \tilde{\gamma}^{i j} \beta^{k}{ }_{, k j}-\tilde{\Gamma}^{j} \beta_{, j}^{i}+\frac{2}{3} \tilde{\Gamma}^{i} \beta^{j}{ }_{, j}+\beta^{j} \tilde{\Gamma}_{, j}^{i}-2 \tilde{A}^{i j} \partial_{j} \alpha \\
& -2 \alpha\left(\frac{2}{3} \tilde{\gamma}^{i j} K_{, j}-6 \tilde{A}^{i j}[\ln (\psi)]_{, j}-\tilde{\Gamma}^{i}{ }_{j k} \tilde{A}^{j k}+8 \pi \tilde{\gamma}^{i j} S_{j}\right) .
\end{aligned}
$$

ADM and BSSN are, in their original forms, unconstrained formulations. The Hamiltonian and momentum constraints are not enforced throughout the simulation but only monitored for quality control. Anderson \& Matzner [17] presented a constrained variation of the ADM formalism where the constraints are solved numerically at every time step. They use equation (5) to solve numerically for the conformal factor $\psi$, while the momentum constraint equations (6) are satisfied by the addition of a longitudinal component to the traceless conformal extrinsic curvature $\tilde{A}^{i j}$ as originally outlined by York [20]. We introduced in Paper I the Hamiltonian relaxation technique that draws 
on the same idea of controlling the Hamiltonian constraint violating modes by adjusting $\psi$. HR uses the conformal factor to solve approximately the Hamiltonian constraint (5), bypassing altogether the corresponding evolution equation (17) for $\psi$. Instead of solving equation (5), HR composes an alternative parabolic equation which relaxes $\psi$ towards a solution of the Hamiltonian constraint through an iterative scheme. The equation used in Paper I was of the form

$$
\partial_{t} \psi=\epsilon_{H}\left(\partial_{t} \mathcal{H}+\eta_{H} \mathcal{H}\right)
$$

where $\epsilon_{H}$ and $\eta_{H}$ are fine-tuning parameters. This technique efficiently quenches the development of Hamiltonian constraint violation instabilities, improving the overall quality of BNS simulations.

In this paper we present the momentum constraint relaxation method, which suppresses the development of momentum constraint violation instabilities and works as a complement to HR. MR is based on correcting the traceless conformal extrinsic curvature $\tilde{A}_{i j}$ with a longitudinal component $(\tilde{l w})_{i j}\left[20\right.$. The operator $(\tilde{l w})_{i j}$ is defined as

$$
(\tilde{l})_{i j} \equiv \tilde{D}_{i} w_{j}+\tilde{D}_{j} w_{i}-\frac{2}{3} \gamma_{i j} \tilde{D}^{n} w_{n}
$$

and $w_{i}$ is known as the vector potential. The momentum constraint equations (6) become now a function of $w_{i}$

$$
\mathcal{M}_{i}=\tilde{\gamma}^{k j}\left[\tilde{D}_{k}(\tilde{l w})_{i j}+6(\tilde{l w})_{i j} \tilde{D}_{k} \ln \psi\right]+\rho_{i}=0
$$

where $\rho_{i}$ collects all terms independent of the vector potential

$$
\rho_{i} \equiv \tilde{\gamma}^{k j}\left[\tilde{D}_{k} \tilde{A}_{i j}+6 \tilde{A}_{i j} \tilde{D}_{k} \ln \psi\right]-\frac{2}{3} \tilde{D}_{i} K-8 \pi S_{i}
$$

Equations (14) form a set of coupled elliptic equations for the components of the vector potential $w_{i}$ that can be solved numerically using a number of different algorithms. Note a formal difference between HR and MR: HR replaces the BSSN evolution equation for the conformal factor (17) with a new equation (12), while MR adds three new auxiliary fields, the $w_{i}$ components, with their corresponding equations (14).

The components of the vector potential $w_{i}$ and the conformal factor $\psi$ are added to the list of dynamical fields evolved using BSSN: $\tilde{\gamma}_{i j}, \tilde{A}_{i j}, K$, and $\tilde{\Gamma}^{i}$. It is important to note that the fields $\tilde{A}_{i j}$ are not updated using the $w_{i}$ new values. Instead, the $w_{i}$ are kept as dynamical variables that evolve from one time step to the next. The r.h.s. of the BSSN evolution equations are modified to include the contribution of $w_{i}$; in equations (8[11), $\tilde{A}_{i j}$ is replaced by $\tilde{A}_{i j}+(\tilde{l w})_{i j}$. This facilitates the implementation of $w_{i}$ boundary conditions, as explained in section 2.3.1.

The work presented in this paper is based on a Successive Under Relaxation algorithm (SUR) similar to the one described in [27]. This algorithm updates the value of $w_{i}$ at each relaxation step $r$ with a correction term

$$
w_{i}^{(r)}=w_{i}^{(r-1)}-\omega_{M} \Delta w_{i}^{(r-1)},
$$

where $\omega_{M}$ is a relaxation parameter such that $0<\omega_{M}<2$ [27]. When $\omega_{M}>1$ (i.e., Successive Over Relaxation or SOR) the method converges faster to the numerical 
solution than for the case $\omega_{M}<1$. However, a key concept behind momentum relaxation is to use the vector potential $w_{i}$ to gradually project the simulation onto the space of solutions of the momentum constraint equations. Since we are interested in gentle updates of $w_{i}$, we use values of $\omega_{M}<1$. SUR also allows for a rather straightforward implementation of the boundary conditions for the vector potential described in section 2.3 .1 .

Their values are updated at each one of the steps of the Iterative Crank-Nicholson (ICN) method (one Predictor and two Corrector steps) used for time integration. The implementation of momentum relaxation, which in this paper is used in combination with HR, is as follows :

1) Initial update of $w_{i}$ : After the initial data set corresponding to a BNS system in circular orbit is read, $w_{i}$ undergoes a relatively large number of relaxation steps (typically about 90), starting from the initial guess $w^{i}=0$. The components are relaxed sequentially in the order $w^{z}, w^{y}$, and $w^{x}$. Given that the three equations are coupled in the components of $w_{i}$, the last component to be relaxed experiences the smoother update. We choose the $x$ component last because the violation of this component of the momentum constraint is the largest due to our choice of coordinate axis (binary aligned along the $y$ axis and $z=0$ the orbital plane).

2) At the Predictor stage of the ICN integration, we perform the update of the fields in the following order:

i) Update of the gravitational fields $\tilde{\gamma}_{i j}, \tilde{A}_{i j}, K$, and $\tilde{\Gamma}^{i}$.

ii) Update of the conformal factor $\psi$ using HR (Paper I).

iii) Update of the vector potential components $w^{z}, w^{y}$, and $w^{x}$. The sources of the elliptic equations are calculated using the values of the fields corresponding to the previous time step. Each equation undergoes typically from 10 to 20 relaxation steps. The boundary conditions for $w_{i}$ are updated right after each relaxation step (section 2.3.11).

iv) Update of the hydrodynamical fields, the lapse function, and the shift vector.

The boundary conditions are updated at the same time as their corresponding fields. The same steps are followed in the first Corrector (second Corrector) stage, but replacing the field values from the previous time step with the corresponding updates generated in the Predictor (first Corrector) step.

\subsection{Lapse and Shift Equations}

We use the same lapse function and shift vectors employed in the development of HR (Paper I): the K-Driver algorithm [26] for $\alpha$ and the shift vector remains unchanged from its initial value ( $\beta$-freezing). All the simulations presented in this paper were performed in the frame that rotates with the binary (corotating), given its superior stability properties over inertial frames [3, 6, 28. We refer the reader to Paper I for the 
details of the numerical implementation as well as the boundary conditions.

\subsection{Boundary Conditions}

We adopt Sommerfeld (radiative) boundary conditions for the conformal metric $\tilde{\gamma}_{i j}$, the traceless part of the extrinsic curvature $\tilde{A}_{i j}$, and the trace of the extrinsic curvature $K$, and we set $\tilde{\Gamma}^{i}=0$ at the boundaries [3]. The boundary conditions for the conformal factor $\psi$ are such that enforce the satisfaction of $\mathcal{H}=0$ in its finite-difference form. They are essential for the control of the Hamiltonian constraint violating modes coming from the grid boundaries.

2.3.1. Boundary Conditions for the Vector Potential $w_{i}$ The boundary conditions for the vector potential $w_{i}$ are inspired in their conformal factor counterparts and also aim at controlling as effectively as possible the incoming constraint violating modes. The boundary conditions for the vector potential $w_{i}$ are such that they enforce the satisfaction to round-off error of the momentum constraint at the grid points next to the boundaries. Equation (14) can be expanded into

$$
\begin{aligned}
& \tilde{\gamma}^{k j}\left[\tilde{D}_{k}(\tilde{l w})_{i j}+6(\tilde{l w})_{i j} \tilde{D}_{k} \ln \psi\right]+\rho_{i}= \\
& \tilde{\gamma}^{k j}\left[\tilde{D}_{k} \tilde{D}_{i} w_{j}+\tilde{D}_{k} \tilde{D}_{j} w_{i}\right]-\frac{2}{3} \tilde{D}_{i} \tilde{D}^{n} w_{n}+6 \tilde{\gamma}^{k j} \partial_{k}(\ln \psi)\left[\tilde{D}_{i} w_{j}+\tilde{D}_{j} w_{i}-\frac{2}{3} \tilde{\gamma}_{i j} \tilde{D}^{n} w_{n}\right] \\
& +\rho_{i}=0 \text {, }
\end{aligned}
$$

where the first and second covariant derivatives of $w_{i}$ are, as usual, functions of the partial derivatives with respect to the spatial coordinates. These partial derivatives of the vector potential are approximated in our finite-difference scheme by second order stencils of the form

$$
\begin{aligned}
& \partial_{x} w_{i}^{n} \sim \frac{w_{i(N, j, k)}^{n}-w_{i(N-2, j, k)}^{n}}{2 \Delta x} \\
& \partial_{x} \partial_{x} w_{i}^{n} \sim \frac{w_{i(N, j, k)}^{n}-2 w_{i(N-1, j, k)}^{n}+w_{i(N-2, j, k)}^{n}}{(\Delta x)^{2}} \\
& \partial_{x} \partial_{y} w_{i}^{n} \sim \frac{w_{i(N, j+1, k)}^{n}-w_{i(N-2, j+1, k)}^{n}-w_{i(N, j-1, k)}^{n}+w_{i(N-2, j-1, k)}^{n}}{4 \Delta x \Delta y} \\
& \text { etc }
\end{aligned}
$$

where $n$ is the relaxation iteration step, $\Delta x$ and $\Delta y$ the grid spacing along the $x$ and $y$ axis, respectively. The above formulae have been written for the grid points next to the boundary $x=x_{\max }$ (i.e.; the points with indices $\left.(N-1, j, k)\right)$. When we replace the differential operators of equation (17) with the finite-difference formulae (18) and solve for the boundary values of $w_{i}$ at the boundary $x=x_{\max }$, we obtain

$$
w_{i(N, j, k)}^{n}=F_{i(N, j, k)},
$$

where $F_{i(N, j, k)}$ are algebraic expressions which depend on $w_{i(N-1, j, k)}^{n}, w_{i(N, j-1, k)}^{n}$, $w_{i(N, j+1, k)}^{n}, w_{i(N, j, k-1)}^{n}$, and $w_{i(N, j, k+1)}^{n}$. The linear system of equations (19) is quite large even for modest-size grids and thus very time consuming to solve for. To expedite 
the process, we evaluate the equations (19) using the newly calculated values at the boundaries as soon as they are ready (i.e., $w_{i(N-1, j, k)}^{n}, w_{i(N, j-1, k)}^{n}$, and $\left.w_{i(N, j, k-1)}^{n}\right)$ and use the previous iteration values for the remainder (i.e., $w_{i(N, j+1, k)}^{n-1}$ and $\left.w_{i(N, j, k+1)}^{n-1}\right)$. This approximation is justified by the fact that these boundaries are evaluated many times in every time step.

Similar to the case of the boundary conditions for the conformal factor, a problem arises at the edges of the grid, where the expression for the boundary value $w_{i(N, N, k)}^{n}$ is now a function of the inverse of the spatial conformal metric $\tilde{\gamma}_{x y}$, which is null at the initial time step. For these points we use bilinear interpolation until the value of $\tilde{\gamma}_{x y}$ crosses some predetermined value which, for the simulations of this paper, is $10^{-6}$.

\subsection{Hydrodynamical Formulation and Initial Data sets}

The numerical simulation of neutron star spacetimes requires algorithms for the evolution of the matter fields. The fluid in the stars is described by a perfect-fluid stress-energy tensor and a polytropic equation of state with constant $\Gamma=2$ is assumed. We use the hydrodynamical methods described in Paper I. The initial state of the BNS in circular orbit is obtained using the Wilson-Mathews Conformally Flat Condition (CFC) approach [29, 30], using the elliptic solver described in 31].

We tested the numerical implementation of the momentum relaxation method using short trial-and-error runs that consisted essentially in BNS simulations performed in small low-resolution grids. To simplify even further these runs, they were based on an initial data set corresponding to a corotating (tidally-locked) binary. The satisfactory performance of these short runs was followed by longer runs based on larger grids and higher resolution. These long runs were also based on more realistic irrotational (zerospin) BNS systems. The details of the initial data sets used in these runs is given in table I of Paper I. The number of points in the short run grid is about 73 times smaller than that of the long run simulations, making it possible to simulate a BNS orbital

period in a couple of hours on a typical single-processor workstation. The long runs were performed using the IA-64 Linux Beowulf cluster Mercury at NCSA.

Our simulations are performed in Cartesian grids and use finite-difference second order operators within grids that have uniform and identical spacing along each axis. We work in the reference frame that rotates with the binary, and the stars are aligned with the $y$ axis.

\section{Results}

\subsection{Short Trial-and-Error Runs}

The free parameters of $\mathrm{MR}$ are the relaxation parameter $\omega_{M}$ and the number of relaxation steps. Their corresponding values were determined empirically and set to $\omega_{M}=0.1$ with 20 relaxation steps for the short runs. Note that $\psi$ and $w_{i}$ are relaxed three times at every time step (i.e., in the ICN predictor and the two corrector stages). 
Figure 1. Short Runs: Evolution of the three components of the momentum constraint residual during the first steps of the time evolution. The upper (lower) plot corresponds to a run without (with) momentum relaxation. The thick line is the initial residual. The curves are plotted following the line with coordinates $(0, y, 0)$, that runs through the center of the star. The companion star, located on the negative $y$ hemisphere, is not shown.
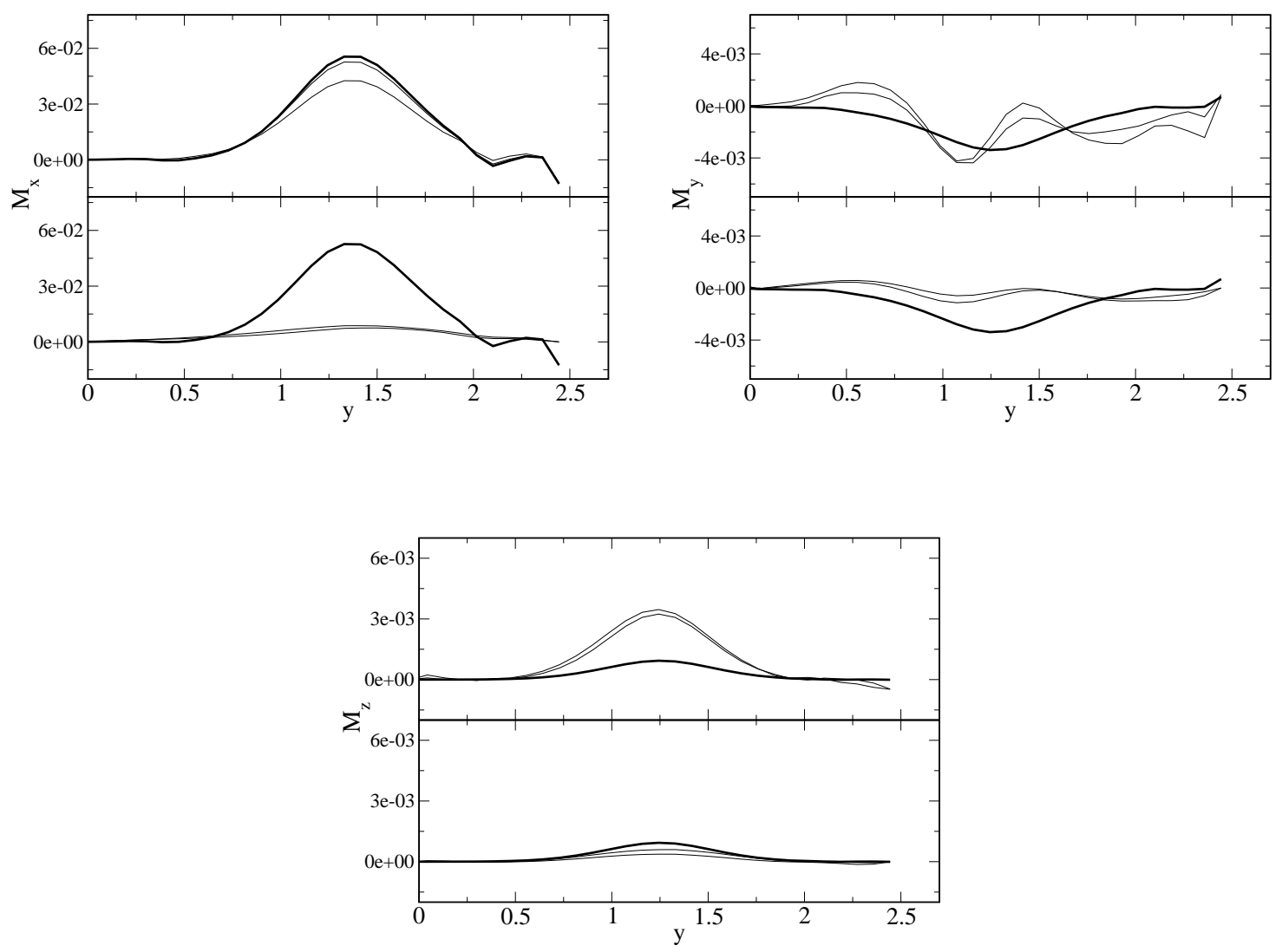

Following the HR method used for the short runs of Paper I, we use the parameter values $\epsilon_{H}=0.0001$ and $\eta_{H}=70.0$, and the relaxation of $\psi$ is performed until the $L_{2}$ norm of the Hamiltonian residual is smaller than the one at the previous time step for up to a maximum of 25 iterations.

For comparison, we show in this and the next section curves corresponding to runs performed using only the BSSN formulation as described in [3], runs using only HR, and runs using both HR and MR. The BSSN runs differ from the HR and HR+MR runs in two aspects. One is that the BSSN runs use the $\Gamma$-Driver [32, 3] while the HR only and $\mathrm{HR}+\mathrm{MR}$ runs use the $\beta$-freeze condition for the shift vector. The other difference is that we use fall-off Robin-like boundary conditions for the lapse function for the BSSN runs and frozen values for the HR and HR+MR runs (see Paper I). All the runs (short and long) have Courant factors of 0.46 . We also conserve the K-Driver and $\Gamma$-Driver (BSSN runs only) parameters, which are set to $\epsilon_{\alpha}=0.125, \eta_{\alpha}=0.1, \epsilon_{\beta}=0.0005, \eta_{\beta}=0.2$, 
Figure 2. Short Runs: Total angular momentum $J$ as a function of time, given as fraction of the orbital period $P . \quad J$ is normalized to its initial value $J_{0}$. The lines correspond to runs using BSSN (dotted), HR only (thin solid), and HR + MR (thick solid).

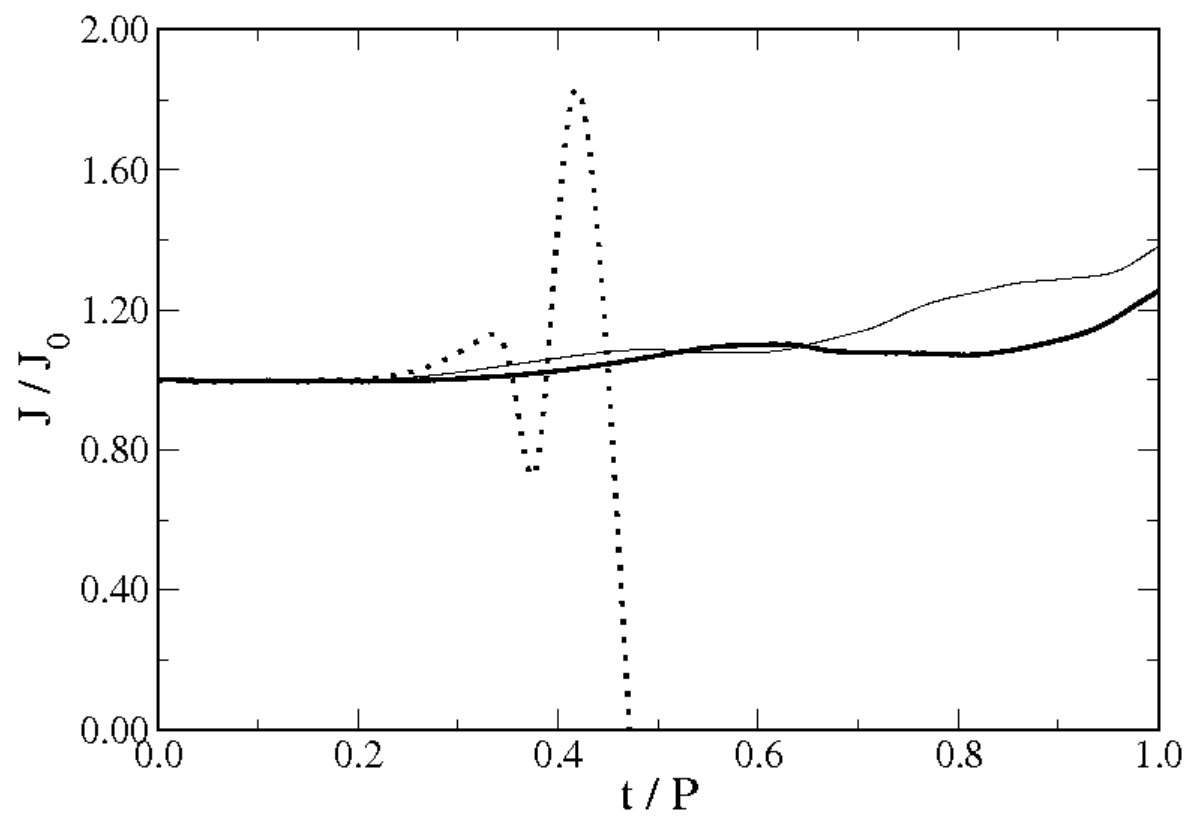

respectively. The K-Driver ( $\Gamma$-Driver) relaxation is iterated 5 (10) times.

Figure 1 presents the evolution of the momentum constraint residuals $\mathcal{M}_{i}$ for the first three time steps of a BNS simulation without (with) MR in the upper (lower) part of the plots. The momentum constraint violation is plotted along the binary axis $(y)$. Only one star is present in the grid (the companion star is in the $y<0$ hemisphere) and its center is at $y \simeq 1$.4. The thick lines correspond in all cases to the initial data. The reduction of the momentum constraint violation caused by MR can be seen both in the bulk of the grid and at the boundary $y=y_{\max }$. Note, however, that this effect is not as dramatic as in the case of $\mathrm{HR}$ and the Hamiltonian constraint violation reduction (compare with figures 1 and 2 of Paper I). Figure 2 shows the evolution of the total angular momentum of the BNS normalized to its initial value. Note that the use of MR (thick solid line) improves the quality of the run only marginally over the performance obtained with HR alone (thin solid line). The small grid size for which and orbital period is roughly equivalent to 10 side-to-side light crossing times makes this result quite remarkable. The BSSN simulation is presented with a dotted line. 
Figure 3. Long Runs: Evolution of the $L_{2}$ norm of the momentum constraint violation across the numerical grid. The solid line corresponds to the $\mathrm{MR}+\mathrm{HR}$ runs, while the dashed curve shows the result of using only HR. The improvement on the satisfaction of the momentum constraint is about a factor of 4 at the end of the simulation.

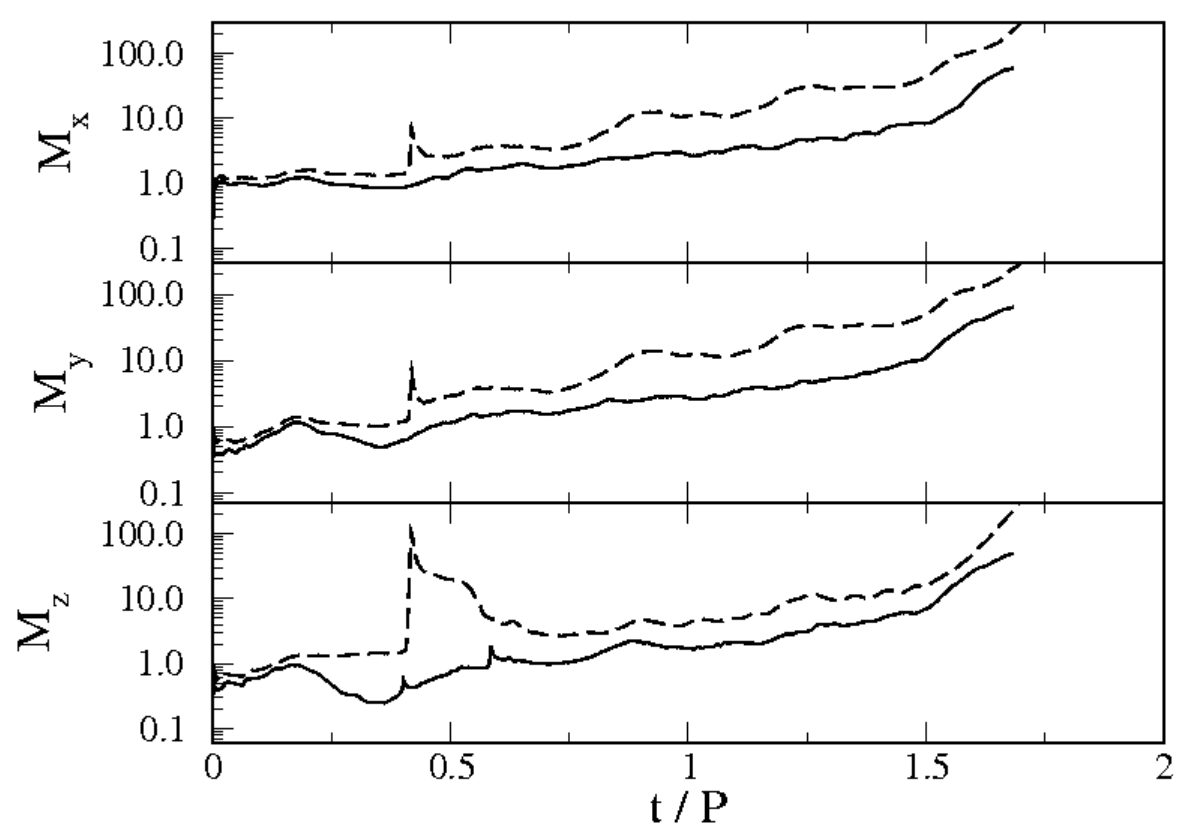

\subsection{Long High-Resolution Large-Grid Runs}

We tested the MR scheme with a large-grid high-resolution simulation of an irrotational BNS system. The details of the initial data set are provided in table 1 of Paper I and the corresponding convergence tests are presented in Appendix A. All the plots of this section show curves that, for clarity, have been normalized to their corresponding initial values. The MR free parameter values used for these long runs were $\omega_{M}=0.01$ with 20 relaxation steps.

The evolution of the $L_{2}$ norm of the three components of the momentum constraint residual $\mathcal{M}_{i}$ across the numerical grid is shown in figure 3. While MR achieves the reduction of the constraint violation as it was designed to do, the improvement is not as impressive as in the case of $\mathrm{HR}$ and the Hamiltonian constraint violation (figure 4 of Paper I). At the end of the simulation, the momentum constraint violation was about four times smaller than in the HR only runs. The spikes present in the HR only curves at $t \simeq 0.4 P$ occur on the stellar surface and are related to matter displacement in the grid, a side-effect of using a frozen shift vector. Note, however, that those spikes disappear when using MR.

Figure 4 shows the evolution of the total angular momentum. The plot shows the 
Figure 4. Long Runs: Evolution of the total angular momentum $J$. The solid, dashed, dash-dotted lines corresponds to the MR+HR, HR only, and BSSN runs respectively. The dotted line shows the PN estimation (see Appendix B of Paper I). The inset expands the plot for the first half orbital period.

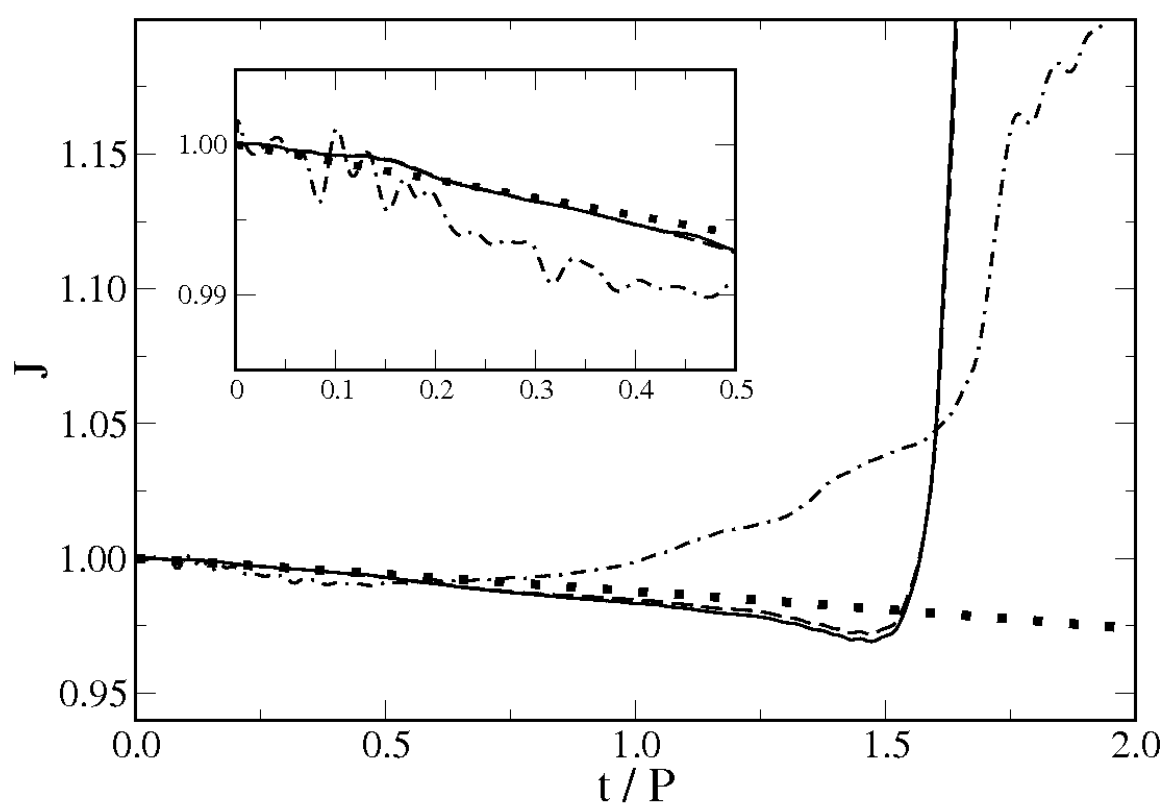

$\mathrm{MR}+\mathrm{HR}$ (solid), HR only (dashed) and BSSN (dashed-dotted) curves, as well as the PN estimation (dotted line) of the angular momentum loss for a point-mass binary with the same mass and angular momentum as the BNS in consideration (see Appendix B of Paper I). Both the HR only and MR $+\mathrm{HR}$ runs agree with the PN prediction for about 1.5 orbital periods. The inset of figure 4 zooms in on the first half of the period, showing the reduced level of noise in the HR only and MR $+\mathrm{HR}$ curves.

The contour plots 5 show in more detail the evolution of the momentum constraint violation during the simulation. The left (right) column shows snapshots of the BSSN $(\mathrm{MR}+\mathrm{HR})$ run at $\mathrm{t}=0,0.5 \mathrm{P}, 1.0 \mathrm{P}$, and $1.5 \mathrm{P}$. The lower surface plots show the rest mass density, highlighting the position of the star in the grid. The instability that eventually stops this simulation originates at the corner of the cubical grid and is related to the use of radiation boundary conditions in combination with a rotating frame. While MR manages to reduce the effect of this constraint violation, simulations based on larger grids will have to address this problem. This effect is less serious in smaller grid runs, where the effect of frame rotation at the corner is consequently smaller. The analysis of this instability as well as a more comprehensive study of shift vectors for use with constraint relaxation methods will be done in future work.

Figure 6] shows the remaining quality control curves: the coordinate separation 
Figure 5. Long Runs: Surface plot of the violation of the $x$ component of the momentum constraint. The left (right) column shows snapshots of the BSSN $(\mathrm{MR}+\mathrm{HR})$ run at $\mathrm{t}=0.5 \mathrm{P}, 1.0 \mathrm{P}$, and $1.5 \mathrm{P}$. The lower surface plots show the rest mass density, indicating the position of the star in the grid.
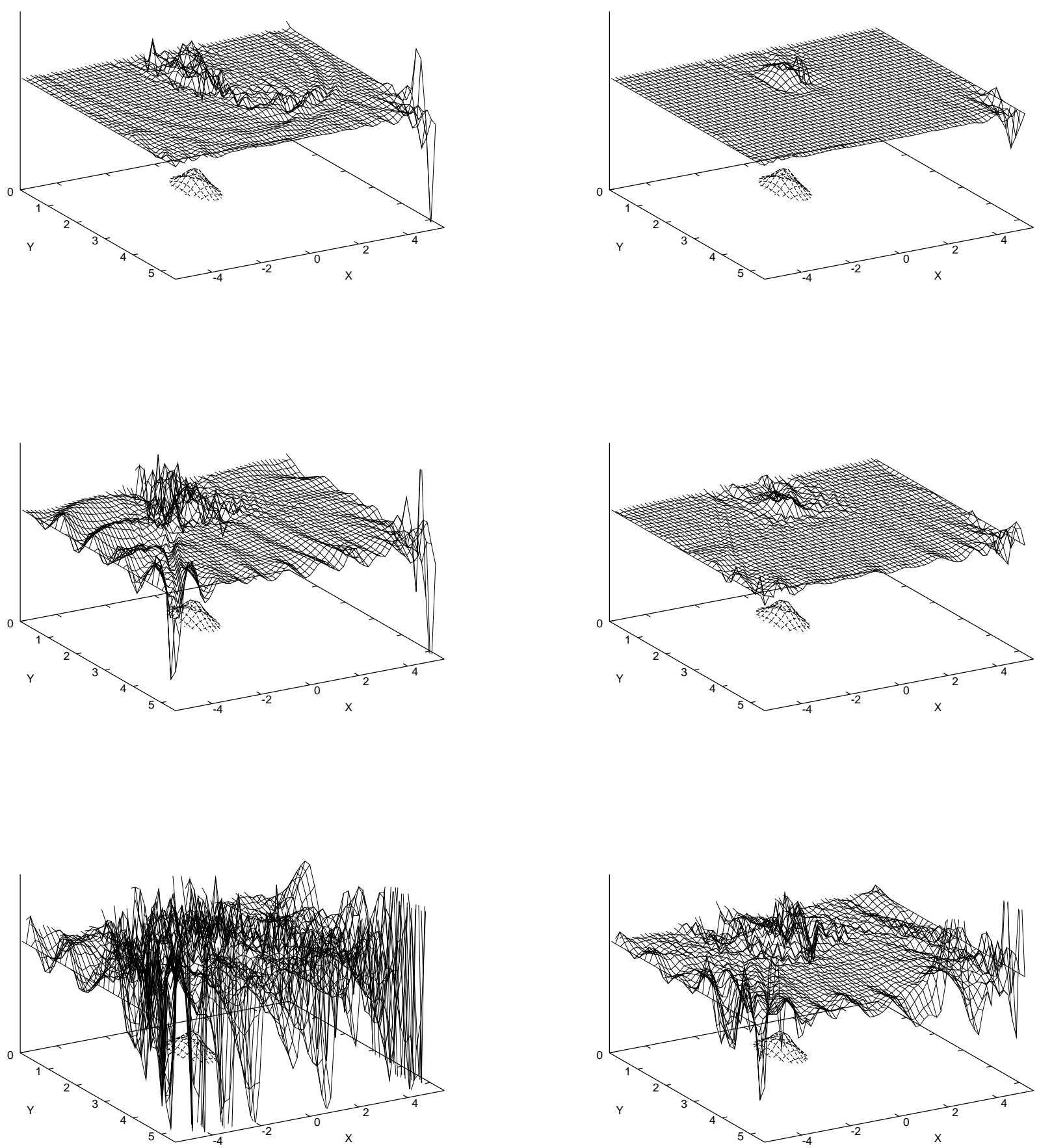
Figure 6. Long Runs: Remaining quality control curves for the MR+HR (solid), HR only (dashed), and BSSN (dash-dotted) runs. From top to bottom, we show the evolution of the coordinate separation between stellar centers $d$, the total gravitational $M$ mass, and the $L_{2}$ of the Hamiltonian constraint.

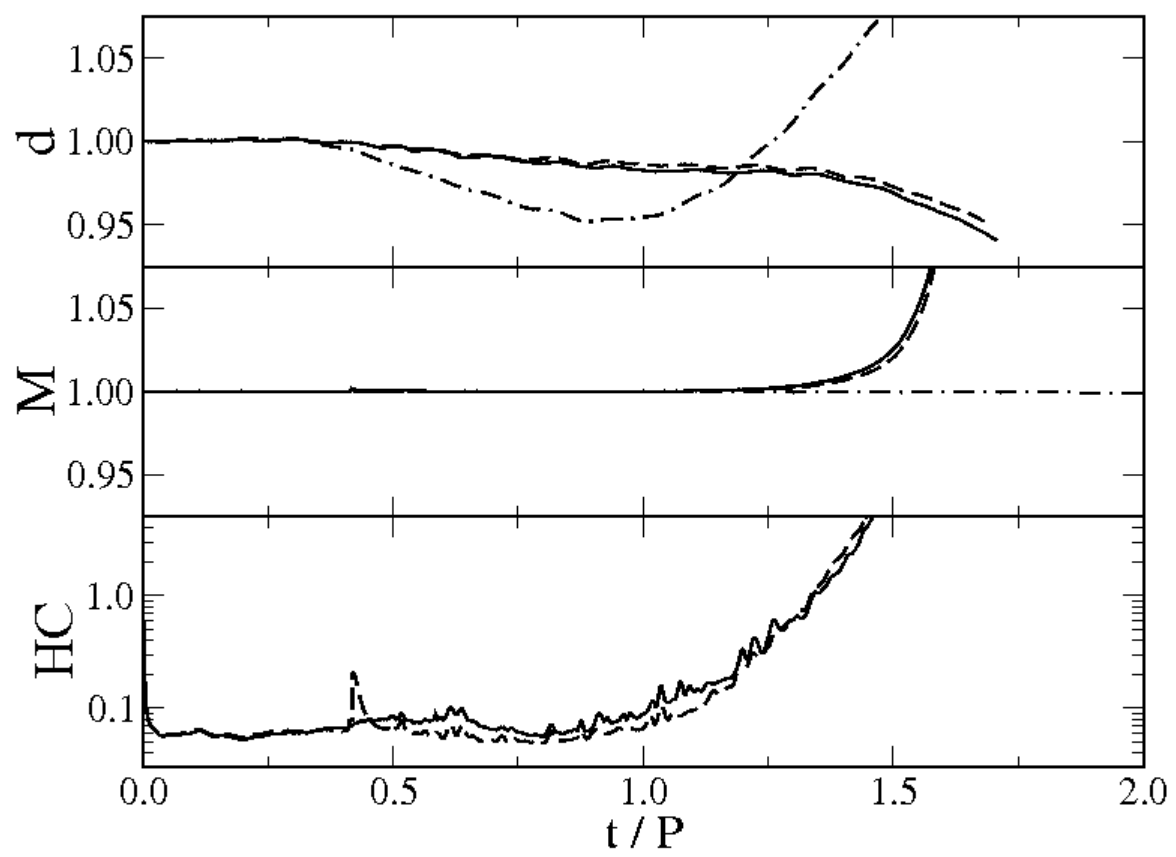

between stellar centers $d$, the total gravitational $M$ mass, and the $L_{2}$ of the Hamiltonian constraint. The latter curve corresponding to the BSSN run is not shown, given that it is out of scale. The total rest mass of the system remains invariant to within a $0.1 \%$ in all runs.

\section{Conclusions}

We introduced the momentum relaxation method which complements the Hamiltonian relaxation scheme presented in Paper I. These algorithms gently relax the conformal factor $\psi$ and the vector potential $w_{i}$ to achieve a gradual control on the growth of constraint violating modes. This smooth updating of the $\psi$ and $w_{i}$ is a key ingredient of the methods since fully enforcing the satisfaction of the constraints (i.e. by relaxing the fields to obtain numerical solutions of the constraint equations) quickly renders the simulation unstable.

The constraint relaxation methods have been tested in combination with BSSN in the simulation of binary neutron stars. Their use not only effectively quenches the constraint violating modes, but also improves the overall quality of the simulation as seen in the behavior of the total gravitational mass and angular momentum of the 
binary. The simulations in this paper end after a couple of orbits due to two principal reasons: the inadequate use of the frozen shift condition and instabilities generated by the use of radiation (Sommerfeld) boundary conditions for the rest of the gravitational fields. The boundary conditions produce constraint violating modes at the corners of the cubical grid which grow worse with increasing grid size. Future work will concentrate on finding the best gauge choices as well as a way to avoid the problems generated at the corners of the grid. Another important aspect that remains to be studied is the behavior of the relaxation techniques in highly dynamical spacetimes. This will be addressed by studying their use in the simulation of BNS mergers. Finally, it remains to be seeing how these methods will affect the simulation in the presence of black holes. One potential complication could arise when using excision, since this would require the development of inner boundary conditions for the conformal factor and the vector potential. Note, however, that new techniques for black hole evolutions without excision have recently been developed 33, 34. These methods have been succesfully employed in numerical simulations of black hole binaries [33, 34, 35, 36] and may prove to be a more robust platform for the implementation of relaxation techniques.

\section{Acknowledgments}

It is a pleasure to acknowledge Wolfgang Tichy for useful discussions and Melissa Troshinsky for carefully reading the manuscript. This work was partially supported by National Computational Science Alliance under Grants PHY020007N. PHY050010T, and PHY050015N.

\section{Appendix A. Code Tests}

To test the convergence of the results obtained when using the $\mathrm{HR}$ and $\mathrm{MR}$, we performed the long run simulation on three different grids with lengths (in units of total rest mass $)=11.6,14.0$, and 18.6, while keeping the same grid spatial resolution ( 40 grid point across the star). Figure A1 shows the evolution of the total angular momentum as a function of time. We see the convergence of the numerical results towards the PN estimation for point-mass binaries (dotted line). We also notice that the corner instability occurs sooner in the largest grid (solid line); the small grid run (dash-dotted line) stops due to the inadequacy of $\beta$-freeze as a shift vector condition in the presence of matter displacement.

To test the numerical convergence of the relaxation results with the spatial grid resolution, we performed three runs based on the irrotational long run using 20 (low res.), 25 (medium res.), and 40 (high res.) grid points across the star. However, in order to see explicitly the second order convergence we fully relaxed the vector potential $w_{i}$ to achieve numerical solutions of the momentum constraint equations (14). The results are shown in figure A2. One important effect is that the simulations become quickly unstable when using full relaxation in combination with BSSN, the K-Driver lapse function and 
Figure A1. Convergence of the momentum relaxation results with varying grid sizes. The convergence test is based on the long run simulation and the plot shows the behavior of the total angular momentum. The labels for curves are (from smallest grid to largest) dash-dotted, dashed, and solid. All the grids have the same spatial resolution. The dotted line shows the PN estimation.

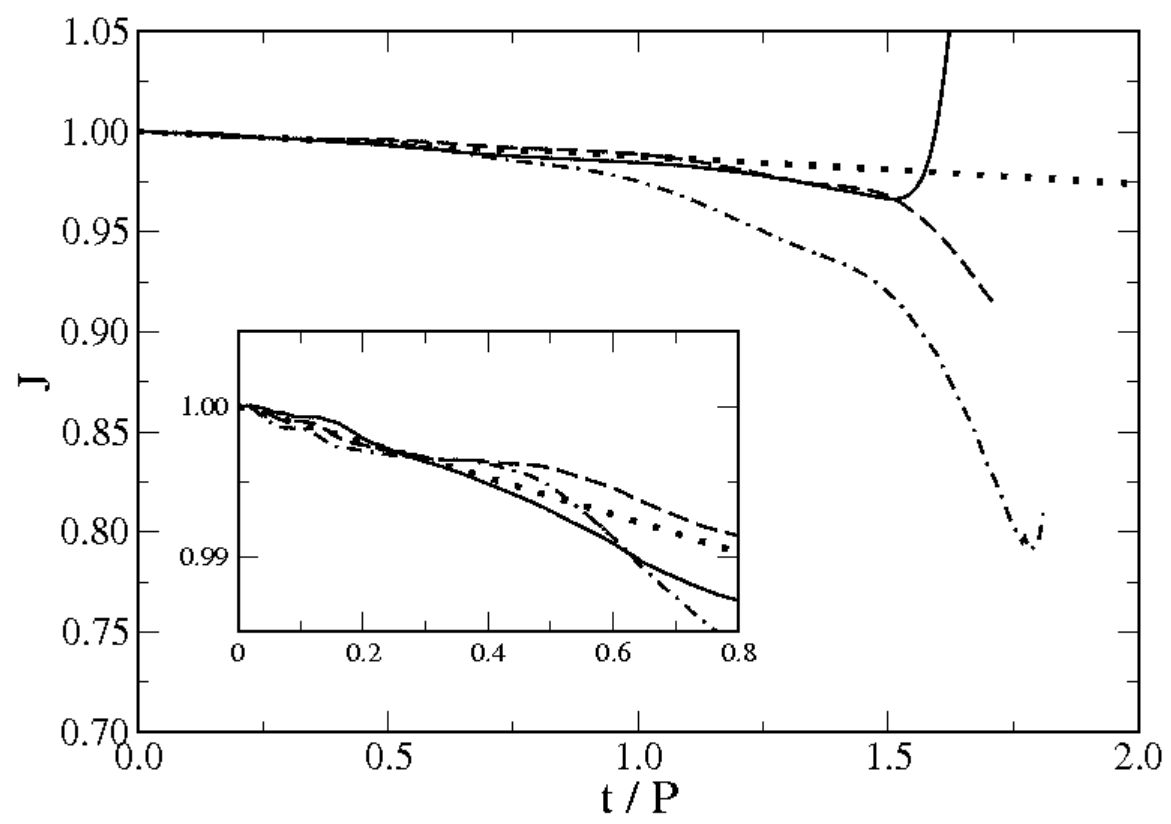

frozen shift vector. In all cases, the runs do not last beyond one tenth of an orbit. The reasons for this incompatibility between BSSN and the full enforcement of constraint satisfaction at every time step are not clear and deserve further study.

The Hamiltonian relaxation technique uses an equation derived from the Hamiltonian constraint (12) to replace the BSSN evolution equation for the conformal factor (17). Since the latter equation is not enforced anymore, we tested its satisfaction by evaluating the $L_{2}$ norm of its violation. We define the violation as

$$
\left\|\partial_{t} \Psi_{B S S N}\right\|_{2} \equiv\left\|\partial_{t} \ln (\psi)-\mathcal{L}_{\beta} \ln (\psi)+\frac{1}{6} \alpha K\right\|_{2}
$$

We implemented the finite-difference version of this equation approximating the time derivative with a second order central difference stencil centered at the time step $t-1$

$\left\|\partial_{t} \Psi_{B S S N}\right\|_{2} \approx\left\|\frac{\ln (\psi)^{t}-\ln (\psi)^{t-2}}{2 \Delta t}-\left(\mathcal{L}_{\beta} \ln (\psi)\right)^{t-1}+\frac{1}{6}(\alpha K)^{t-1}\right\|_{2}$.

Similarly, we tested the violation of the $x x$ component of the traceless extrinsic curvature after the vector potential $w_{i}$ is updated. Calculating $\tilde{A}_{x x}^{N e w}$ as $\tilde{A}_{x x}^{N e w}=\tilde{A}_{x x}+(\tilde{l w})_{x x}$, we 
Figure A2. Evolution of the $L_{2}$ norm of the violation of the components of the momentum constraint for the irrotational Long Run described in table I of Paper I with three different grid resolutions. The dotted, dashed, and solid curves correspond to the low, medium, and high resolution runs and the numerical factors multiplying the curves correspond to the ratios between grid spacings.

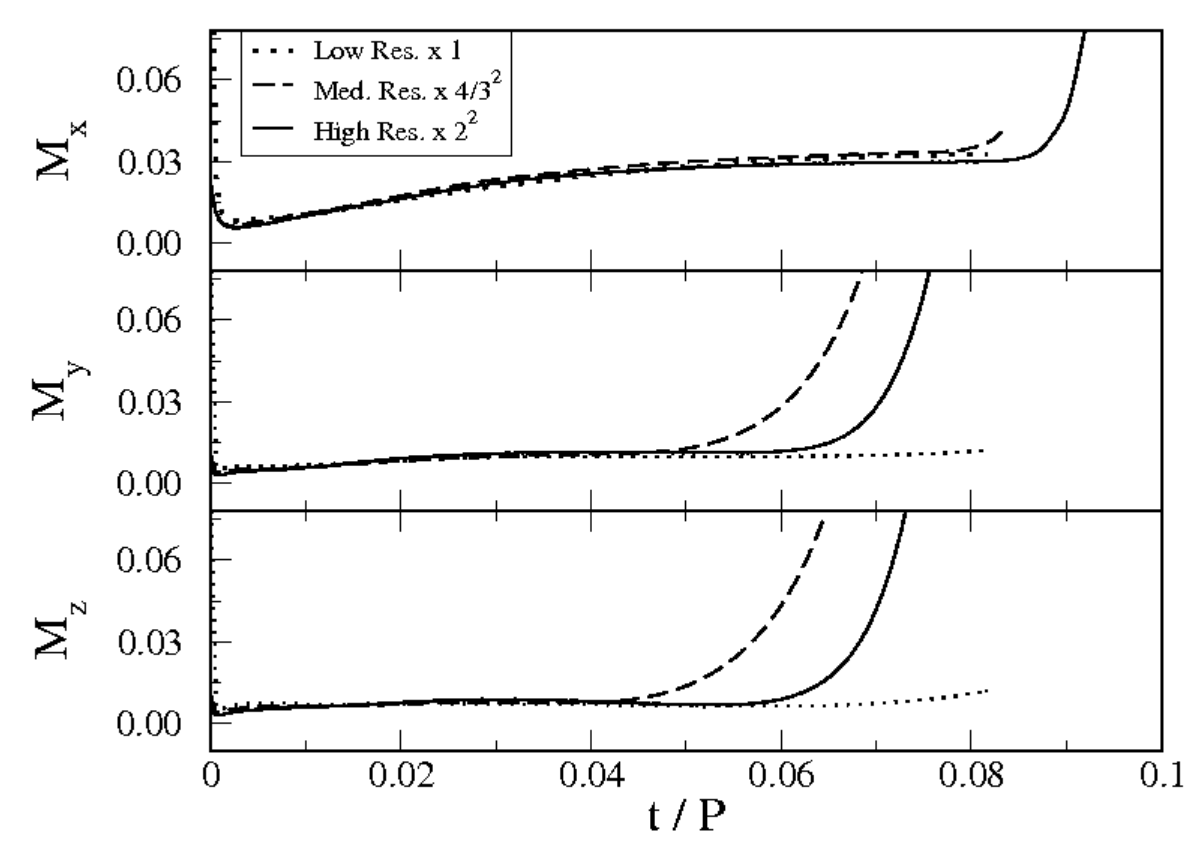

define the violation as

$\left\|\partial_{t} \tilde{A}_{x x B S S N}^{N e w}\right\|_{2} \approx \| \frac{\tilde{A}_{x x}^{N e w ~}{ }^{t}-\tilde{A}_{x x}^{N e w(t-2)}}{2 \Delta t}-\left(\mathcal{L}_{\beta} \tilde{A}_{x x}^{N e w}\right)^{t-1}-\left(\left[\text { rhs } \tilde{A}_{x x}^{N e w}\right]\right)^{t-1} \|_{2}$,

where $\left[r h s \tilde{A}_{x x}^{N e w}\right]$ represents the r.h.s. of equation (10).

Figure A3 shows the behavior of such violations for the small runs described in section 3.1. The comparison of $\left\|\partial_{t} \Psi_{B S S N}\right\|_{2}$ (top) from the HR+MR run (solid curve) with the corresponding violation from the BSSN run (dashed curve) shows no significant difference $\ddagger$. The plot of $\left\|\partial_{t} \tilde{A}_{x x B S S N}^{N e w}\right\|_{2}$ (bottom) reflects a drift in the BSSN curve that is not present in the HR+MR case. This apparent "improvement" due to the use of relaxation methods is likely due to the overall stability gained throughout the simulation and not a specific betterment of the satisfaction of equation (10).

$\ddagger$ Note that the BSSN curve is not identically zero since the second order convergence in time is achieved in the evolution through the use of ICN integration and not the time derivative stencil of equation $\mathrm{A} .2$ 
Figure A3. Evolution of the $L_{2}$ norm of the violation of the BSSN evolution equation for the conformal factor (top) and the $x x$ component of the traceless extrinsic curvature (bottom). The dashed and solid curves correspond to the BSSN and HR+MR short runs described in section 3.1

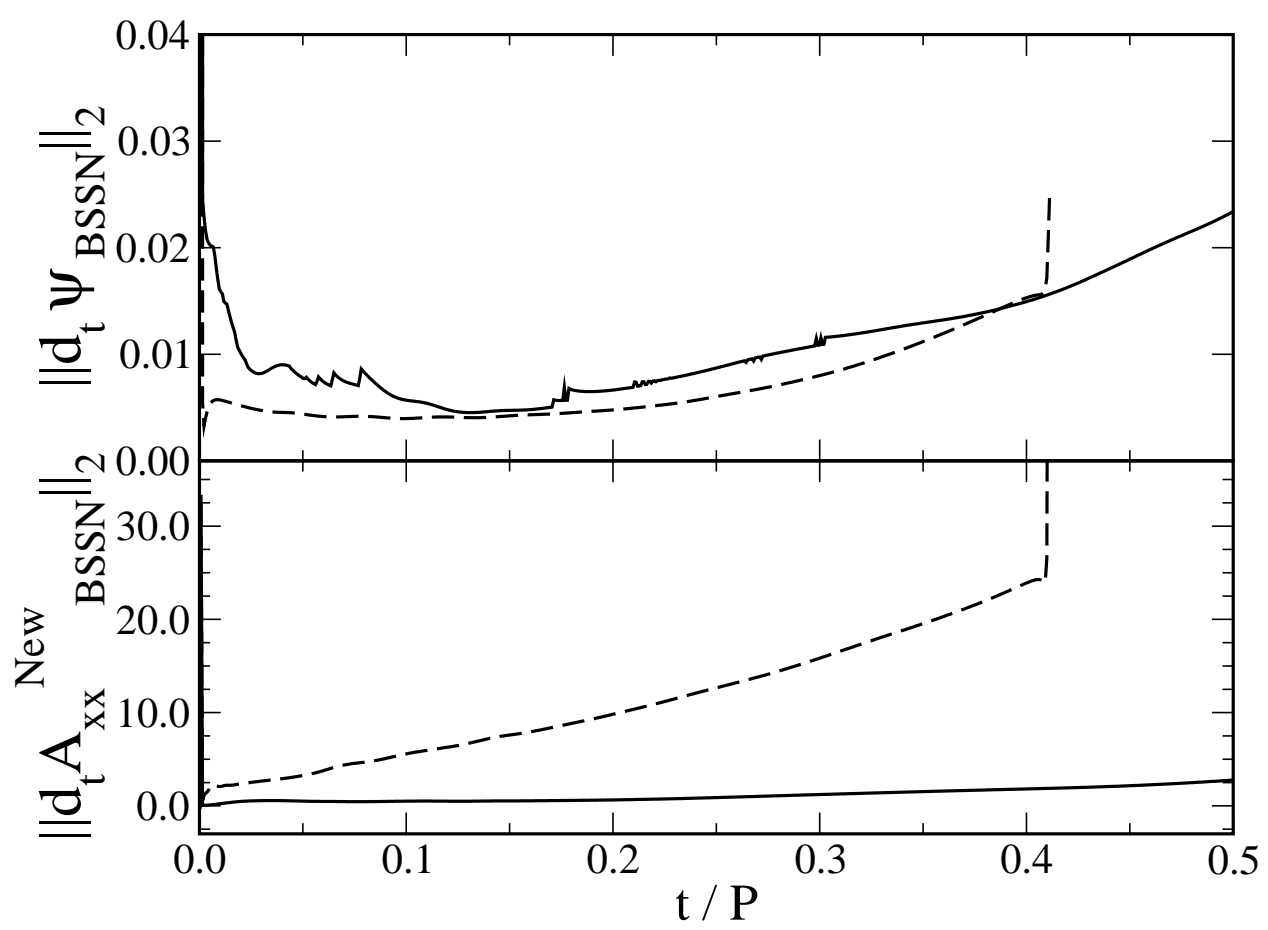

[1] M. Shibata and K. Uryu, Phys. Rev. D 61, 064001 (2000).

[2] M. Shibata and K. Uryu, Prog. Theor. Phys. 107, 265 (2002).

[3] M. D. Duez, P. Marronetti, S. L. Shapiro, and T. W. Baumgarte, Phys. Rev. D 67, 024004 (2003).

[4] M. Shibata, K. Taniguchi, and K. Uryu, Phys. Rev. D 68, 084020 (2003).

[5] M. Miller, P. Gressman and W. M. Suen, Phys. Rev. D 69, 064026 (2004).

[6] P. Marronetti, M. D. Duez, S. L. Shapiro, and T. W. Baumgarte, Phys. Rev. Lett. 92, 141101 (2004).

[7] M. Kawamura, K. i. Oohara and T. Nakamura, astro-ph/0306481

[8] E. Evans, S. Iyer, E. Schnetter, W. M. Suen, J. Tao, R. Wolfmeyer, and H. M. Zhang, Phys. Rev. D 71, 081301 (2005).

[9] R. Arnowitt, S. Deser and C. W. Misner, in Gravitation: An Introduction to Current Research, edited by L. Witten (Wiley, New York, 1962).

[10] M. Shibata and T. Nakamura, Phys. Rev. D 52, 5428 (1995).

[11] T. W. Baumgarte and S. L. Shapiro, Phys. Rev. D,59, 024007 (1999).

[12] M. W. Choptuik, Phys. Rev. Lett. 70, 9 (1993).

[13] A. M. Abrahams, G. B. Cook, S. L. Shapiro, and S. A. Teukolsky, Phys. Rev. D 49, 5153 (1994).

[14] M. W. Choptuik, E. W. Hirschmann, S. L. Liebling, and F. Pretorius, Class. Quant. Grav. 20, 1857 (2003). gr-qc/0301006

[15] M. W. Choptuik, E. W. Hirschmann, S. L. Liebling, and F. Pretorius, Phys. Rev. D 68, 044007 (2003). gr-qc/0301006

[16] S. Bonazzola, E. Gourgoulhon, P. Grandclement, and J. Novak, Phys. Rev. D 70, 104007 (2004).

[17] M. Anderson and R. A. Matzner, Found. of Physics 35, No. 9 (2005). gr-qc/0307055 
[18] P. Marronetti, Class. Quant. Grav. 22, 2433 (2005). gr-qc/0501043

[19] J. W. York. J. Math. Phys. 14456 (1973).

[20] J. W. York, Jr., in Sources of Gravitational Radiation, edited by L . Smarr (Cambridge Univ. Press, Cambridge, England, 1979), p. 83.

[21] R. A. Matzner, M. F. Huq, and D. Shoemaker, Phys. Rev. D 59, 024015 (1999). gr-qc/9805023.

[22] P. Marronetti, Mijan F. Huq, P. Laguna, Luis Lehner, Richard A. Matzner, and D. Shoemaker. Phys. Rev. D 62024017 (2000). gr-qc/0001077.

[23] P. Marronetti and Richard A. Matzner. Phys. Rev. Lett. 855500 (2000). gr-qc/0009044

[24] H. P. Pfeiffer, G. B. Cook, and S. A. Teukolsky. Phys. Rev. D 66024047 (2002).

[25] E. Bonning, P. Marronetti, D. Neilsen, and R. Matzner, Phys. Rev. D 68, 044019 (2003). gr-qc/0305071.

[26] J. Balakrishna, G. Daues, E. Seidel, W.-M. Suen, M. Tobias, and E. Wang, Class. Quantum Grav. 13, L135 (1996).

[27] W. H. Press, S. A. Teukolsky, W. T. Vetterling, and B. P. Flannery. Numerical Recipes: The Art of Scientific Computing (Cambridge University Press, Cambridge, England, 1992), section 19.5.

[28] F. D. Swesty, E. Y. Wang, and A. C. Calder, Astrophys. J. 541, 937 (2000).

[29] J. R. Wilson and G. J. Mathews, Phys. Rev. Lett. 75, 4161 (1995).

[30] J. R. Wilson, G. J. Mathews, and P. Marronetti, Phys. Rev. D 54, 1317 (1996).

[31] P. Marronetti and S. L. Shapiro, Phys. Rev. D 68, 104024 (2003).

[32] M. Alcubierre and B. Brügmann, Phys. Rev. D 63, 104006 (2001).

[33] M. Campanelli, C. O. Lousto, P. Marronetti and Y. Zlochower, arXiv:gr-qc/0511048

[34] J. G. Baker, J. Centrella, D. I. Choi, M. Koppitz and J. van Meter, arXiv:gr-qc/0511103

[35] F. Herrmann, D. Shoemaker and P. Laguna, arXiv:gr-qc/0601026.

[36] M. Campanelli, C. O. Lousto and Y. Zlochower, arXiv:gr-qc/0601091. 


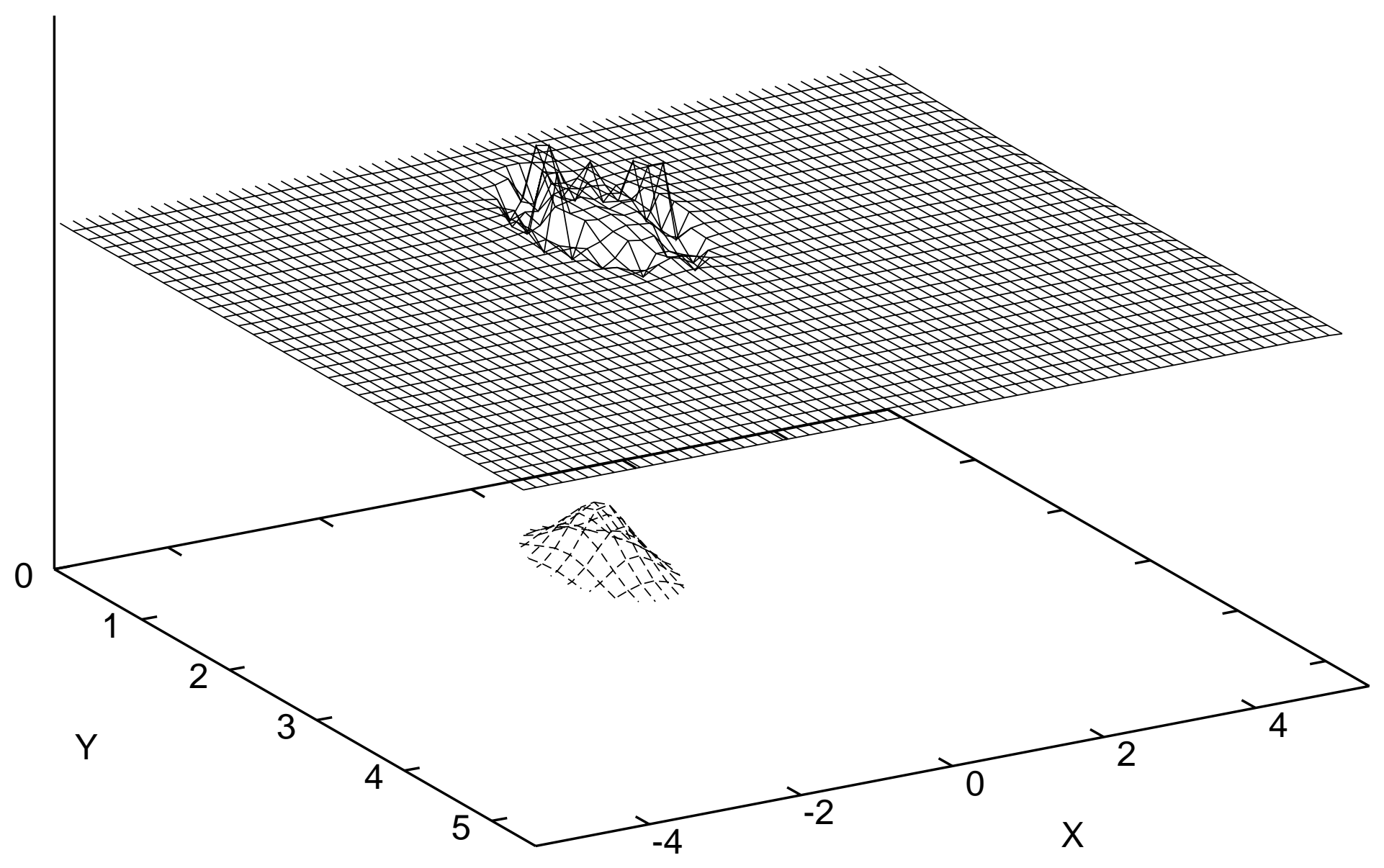




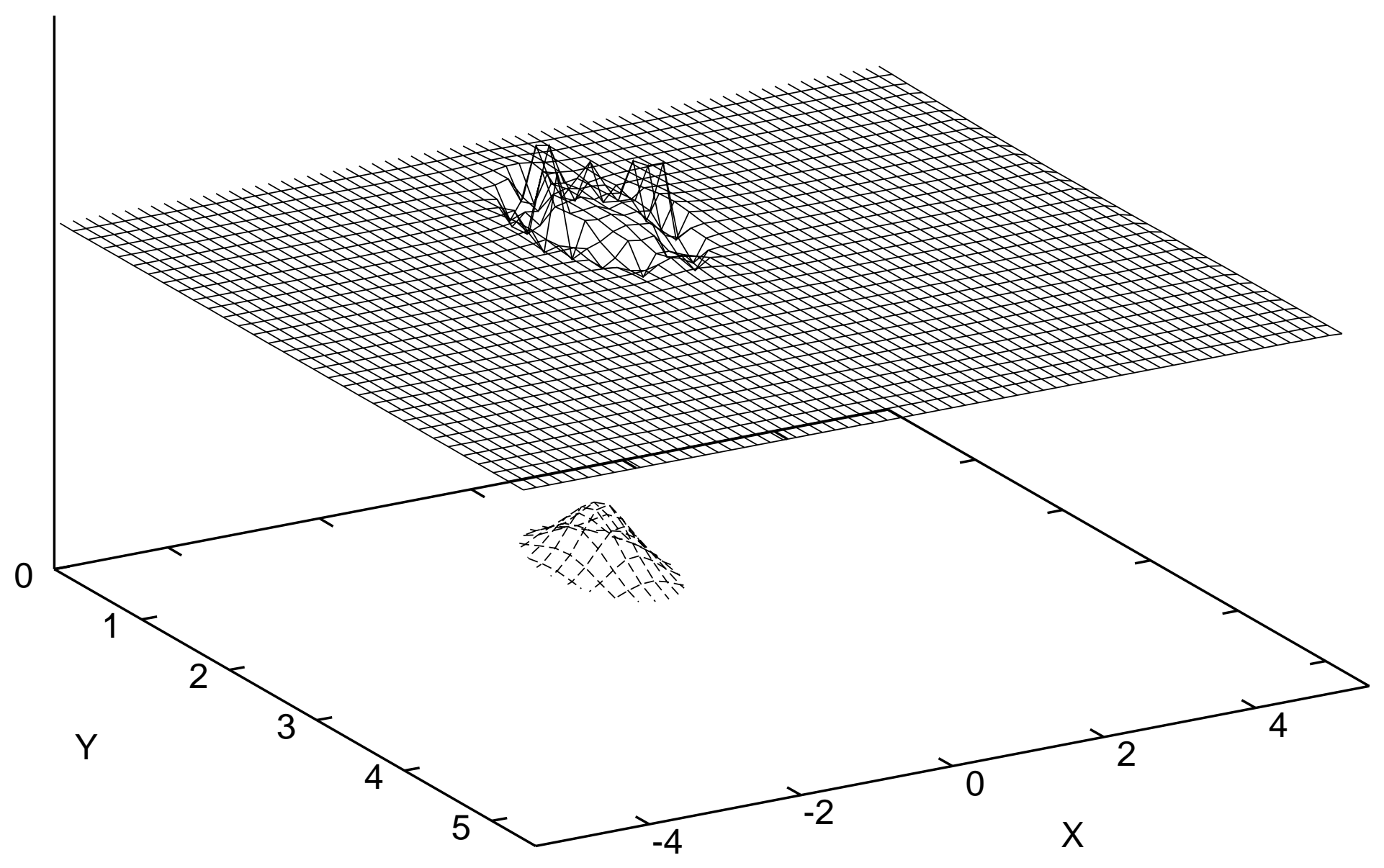

